\title{
Production of the Fimbrial Adhesin 987P by Enterotoxigenic Escherichia coli during Growth under Controlled Conditions in a Chemostat
}

\author{
By MARJAN W. VAN DER WOUDE, ${ }^{*}$ FRITS K. DE GRAAF \\ AND HENK W. VAN VERSEVELD \\ Department of Microbiology, Biological Laboratory, Vrije Universiteit, De Boelelaan 1087, \\ 1081 HV Amsterdam, The Netherlands
}

(Received 17 April 1989; revised 12 July 1989; accepted 22 August 1989)

\begin{abstract}
The effects of growth conditions on the production of 987P fimbriae by the enterotoxigenic Escherichia coli strain 1592 were examined in steady state chemostat experiments at different specific growth rates. The amount of fimbriae produced by fimbriate cells $\left(\mathrm{P}^{+}\right)$was dependent on the specific growth rate $(\mu)$. Under aerobic growth conditions fimbriae production increased with higher $\mu$ values till $\mu=0.40 \mathrm{~h}^{-1}$ and decreased again at $\mu$ values close to $\mu_{\text {max }}\left(0.48 \mathrm{~h}^{-1}\right)$. Under anaerobic growth conditions the maximal production was comparable to that under aerobic growth conditions, and was also maximal close to $\mu_{\max }\left(0.16 \mathrm{~h}^{-1}\right)$. Phase variation, measured as the percentage of fimbriate cells in a particular population, was independent of $\mu$. The composition of the growth medium influenced both phase variation and overall production of fimbriae. A shift from minimal to a complex medium induced a rapid reduction in the amount of fimbriae per $\mathrm{P}^{+}$cell and a slower reduction in the percentage of $\mathrm{P}^{+}$cells. A shift from complex to minimal medium resulted in an increase in the percentage of $\mathrm{P}^{+}$cells and a constant amount of fimbriae per $\mathrm{P}^{+}$cell. The frequency of the phase switch was calculated for different growth conditions. The frequency of the $\mathrm{P}^{+} \rightarrow \mathrm{P}^{-}$switch between two steady states was $2.7 \times 10^{-2}$. In batch culture the frequency of the $\mathrm{P}^{-} \rightarrow \mathrm{P}^{+}$switch was minimally $2.9 \times 10^{-2}$. The results indicate that phase variation and the production of $987 \mathrm{P}$ fimbriae by fimbriate cells are under independent physiological control.
\end{abstract}

\section{INTRODUCTION}

Fimbriae play an important role in the adhesion of pathogenic Escherichia coli strains to the epithelial cells of their hosts (de Graaf \& Mooi, 1986). The biosynthesis of fimbriae is subject to extensive regulation. Two types of regulation can be distinguished: a quantitative regulation affecting length and number of fimbriae per cell and a qualitative regulation known as phase variation. The latter form of regulation has been defined as the all-or-none oscillation between the fimbriate $\left(\mathrm{P}^{+}\right)$and non-fimbriate phase $\left(\mathrm{P}^{-}\right)$of individual cells (Brinton, 1959).

Quantitative regulation encompasses the effects of temperature, $\mathrm{pH}$, composition of the growth medium and specific growth rate of the cells on the production of fimbriae. These effects have been studied in detail on the production of K99 and F41 fimbriae by enterotoxigenic $E$. coli strains. Production of such fimbriae is optimal at a $\mathrm{pH}$ of 7.0 and at maximal specific growth rate at $37^{\circ} \mathrm{C}$ (van Verseveld et al., 1985). Most probably, quantitative regulation plays a role in the production of all types of fimbriae, even though the extent of the influence of the different environmental factors may vary with different types of fimbriae.

Phase variation, sometimes described as a qualitative regulation, has been found in connection with a limited number of the different types of fimbriae. The phase variation of type 1 fimbriae has been investigated most extensively. The molecular basis for variation was

Abbreviations: $\mathrm{P}^{+}$, fimbriate phase $\mathrm{P}^{-}$, non-fimbriate phase. 
discovered by the identification of an invertible DNA fragment containing the promoter for the fimbrial genes, indicating that phase variation is under transcriptional control (Abraham et al., 1985; Eisenstein, 1981). Two different proteins are responsible for the inversion of this DNA fragment (Abraham et al., 1985; Klemm, 1986). In addition, type 1 phase variation is dependent on the integration host factor (Dorman \& Higgins, 1987; Eisenstein et al., 1987). Even though the molecular mechanism of phase variation has been elucidated it is not clear how the frequency or the direction of the phase switch is affected by environmental conditions. Considering the fact that particular growth conditions favour one of the two phases, it seems likely that regulation of some kind occurs. That cultures can be enriched for cells in the $\mathrm{P}^{+}$state by growth in static liquid medium might also be interpreted as the result of regulation of the phase variation (Old \& Duguid, 1970), as well as the temporal appearance of different fimbrial antigens during batch cultures (Nowicki et al., 1984, 1985).

987P fimbriae mediate the adhesion of $E$. coli cells to the intestinal epithelial cells of pigs (Nagy et al., 1976). The expression of 987P fimbriae is subject to phase variation as seen by differences in colony morphology on blood-agar plates (Nagy et al., 1977). The fimbriae are morphologically comparable to type 1 fimbriae, being rod-shaped with a diameter of about $6 \mathrm{~nm}$ and having an apparent axial hole (Gaastra \& de Graaf, 1982). They consist of similar repeating protein subunits. The gene encoding the subunit has been cloned and sequenced (de Graaf \& Klaasen, 1986, 1987). The molecular mass of the subunit as deduced from the nucleotide sequence is $17184 \mathrm{Da}$ with a 21 or 23 amino acid signal-sequence (de Graaf \& Klaasen, 1987). In this study we aimed to analyse production of $987 \mathrm{P}$ fimbriae under different growth conditions and to make a distinction between the contribution of both regulatory mechanisms to the total amount of fimbriae produced under the different conditions.

\section{METHODS}

Bacterial strains and media. The 987P-fimbriae-producing Escherichia coli strain 1592, serotype 09:K103:987P was obtained from H. W. Moon (National Animal Disease Center, Ames, Iowa, USA). The strain was grown in minimal medium containing $\left(1^{-1}\right): \mathrm{KH}_{2} \mathrm{PO}_{4}(4.5 \mathrm{~g}) ; \mathrm{K}_{2} \mathrm{HPO}_{4}(7.5 \mathrm{~g}) ; \mathrm{NH}_{4} \mathrm{Cl}(2 \mathrm{~g}) ; \mathrm{MgSO}_{4} .7 \mathrm{H}_{2} \mathrm{O}(0.2 \mathrm{~g})$; $\mathrm{MnCl}_{2} .4 \mathrm{H}_{2} \mathrm{O}(1 \mathrm{mg}) ; \mathrm{FeCl}_{3} \cdot 6 \mathrm{H}_{2} \mathrm{O}(0 \cdot 135 \mathrm{mg}) ; \mathrm{CaCl}_{2} .2 \mathrm{H}_{2} \mathrm{O}(0.4 \mathrm{mg})$, and glucose as carbon and energy source. This medium was supplemented with yeast extract (Oxoid) as indicated. Minca medium was also used; it contained (1-1) $\mathrm{KH}_{2} \mathrm{PO}_{4}(1.36 \mathrm{~g}) \mathrm{Na}_{2} \mathrm{HPO}_{4} .2 \mathrm{H}_{2} \mathrm{O}(10 \cdot 1 \mathrm{~g})$, glucose $(1 \mathrm{~g}), \mathrm{MgSO}_{4} \cdot 7 \mathrm{H}_{2} \mathrm{O}(10 \mathrm{mg}), \mathrm{MnCl}_{2} .4 \mathrm{H}_{2} \mathrm{O}$ $(1 \mathrm{mg}), \mathrm{FeCl}_{3} .6 \mathrm{H}_{2} \mathrm{O}(0.135 \mathrm{mg}), \mathrm{CaCl}_{2} .2 \mathrm{H}_{2} \mathrm{O}(0.4 \mathrm{mg})$, Casamino acids (Difco $\left.1 \mathrm{~g}\right)$ (Guinee et al., 1976); it was supplemented with $1 \%(\mathrm{w} / \mathrm{v})$ yeast extract. Trypticase soy broth (TSB; Oxoid) was used also. For growth of the strain on solid medium either TSB with $1 \%(\mathrm{w} / \mathrm{v})$ agar or blood-agar $(5 \%, \mathrm{v} / \mathrm{v}$, sheep blood in blood agar base; Oxoid) was used. Small grey colonies consist of cells in the fimbriae-positive phase as shown by high $987 \mathrm{P}$ production in ELISA; larger white colonies consist of cells in the negative phase as shown by low 987P production in ELISA (Nagy et al., 1977).

Immunological assays. The amount of 987P fimbriae was determined by (ELISA) as described by de Graaf \& Klaasen (1986). Purified 987P fimbriae, 987P antiserum raised against purified fimbriae and monoclonal antibodies conjugated with horse-radish peroxidase were kindly provided by F. G. van Zijderveld (Central Veterinary Institute, Lelystad, the Netherlands). The purified 987P fimbriae were used as a standard in the ELISA to quantify the amount of fimbriae produced. For determination of the production of fimbriae ultrasonic extracts of cells resuspended in phosphate-buffered saline [PBS; $50 \mathrm{mM}$ phosphate buffer, $\mathrm{pH} 7 \cdot 1,0.9 \%(\mathrm{w} / \mathrm{v}) \mathrm{NaCl}$ ] to an $\mathrm{OD}_{660}$ of 0.5 were used as antigen in the ELISA. Values for $987 \mathrm{P}$ concentrations given are means of duplicate determinations with less than $10 \%$ difference.

The number of fimbriate cells in samples of liquid cultures was determined by an immunofluorescent assay as described by Nowicki et al. (1984) with a few alterations. A conjugate of $\mathrm{IgG}$ and fluorescein isothiocyanate (FITC) was made as described by McKinney et al. (1966). Cells were fixed on a glass-slide by air-drying $10 \mu l$ of culture and then incubating the slide in a fresh solution of $3.5 \%(\mathrm{w} / \mathrm{v})$ paraformaldehyde in PBS for 10 min. The fixed cells were then incubated with the conjugate for $60 \mathrm{~min}$ at room-temperature in a moist chamber. After rinsing twice with PBS the slides were carefully dried and a drop of buffer consisting of $100 \mathrm{mg}$ paraphenylene diamine, $10 \mathrm{ml}$ PBS and $90 \mathrm{ml}$ glycerol was added. Slides were kept in the dark at $-20^{\circ} \mathrm{C}$ until analysis. Fluorescent bacteria in a field were counted in a Leitz phase contrast/fluorescence microscope equipped with an excitation filter of $490 \mathrm{~nm}$ and an emission filter of $510 \mathrm{~nm}$. The total number of bacteria in the same field was determined using phase contrast. Per sample a minimum of 100 individual cells was examined. The standard deviation found between different fields was less than $10 \%$.

Analysis of dry weight. Biomass was determined by the collection of cells on $0.2 \mu \mathrm{m}$, constant weight nitrocellulose filters (Sartorius) followed by drying for a minimum of $2 \mathrm{~h}$ at $105^{\circ} \mathrm{C}$ and weighing. 
Fermenter mode. E. coli strain 1592 was grown in continuous culture in a chemostat at $37^{\circ} \mathrm{C}$ and a controlled $\mathrm{pH}$ of 7.2 as described by Stouthamer \& Bettenhausen (1975). Glucose was added to the minimal medium as the growth-limiting substrate ( $10 \mathrm{~mm}$ for aerobic and $30 \mathrm{~mm}$ for anaerobic conditions). Yeast extract was added to the minimal medium as indicated. TSB was used at a concentration of $6 \mathrm{~g} \mathrm{l}^{-1}$.

For growth in batch cultures $500 \mathrm{ml}$ flasks containing $100 \mathrm{ml}$ medium were incubated at $37^{\circ} \mathrm{C}$ on a shaking platform. Minimal medium used for batch cultures contained $0 \cdot 2 \%$ glucose; TSB was used at a concentration of $30 \mathrm{~g} \mathrm{l}^{-1}$.

Inocula for the chemostat cultures were obtained as follows : a colony of strain 1592 was restreaked repeatedly on TSB agar plates. This resulted in colonies with identical morphology that were 987P-negative as judged by lack of agglutination with $987 \mathrm{P}$ antiserum. Subsequently, a single colony was used to inoculate $100 \mathrm{ml}$ of minimal medium. After the culture reached stationary phase it was used to inoculate the chemostat. These cultures agglutinated with 987P antiserum. Variable percentages of $\mathrm{P}^{+}$bacteria were present in these cultures as judged by colony morphology on blood-agar plates, but always at least $50 \%$ of these colonies were of the typical $\mathrm{P}^{+}$ morphology being small and grey.

Determination of the frequency of the phase switch. The frequency of phase switch was calculated by using the formula $(M / N) / g$ in which $M$ is the number of cells which have changed phase, $N$ is the total number of cells and $g$ is the number of generations in which change is measured (Enomoto \& Stocker, 1975). In the chemostat and batch experiments $M / N$ was determined using the immunofluorescent staining method described above. In the chemostat experiments $g$ is $\ln 2 / \mu$. In the batch experiment $g$ was calculated from the growth curve.

The frequency of phase switch was also determined using the procedure described for the analysis of phase variation with type 1 fimbriae (Eisenstein, 1981). The morphology of colonies grown on blood agar plates was used to determine the $\mathrm{P}^{+}$or $\mathrm{P}^{-}$state. A grey, $\mathrm{P}^{+}$colony was restreaked on a blood-agar plate. Colonies of similar grey morphology were taken to have grown from a single $\mathrm{P}^{+}$cell. A single colony was cut-out of the agar and resuspended in $5 \mathrm{ml}$ PBS. Dilutions were made to determine the total number of bacteria in the colony for obtaining the value for $g$. The ratio of cells in the negative phase to the total number of cells was determined from colony morphology after growth on blood-agar plates. The $\mathrm{P}^{-} \rightarrow \mathrm{P}^{+}$switch-frequency was obtained in the same manner but starting with a white colony.

\section{RESULTS}

From preliminary experiments in which fimbriae production and phase variation were determined during growth in batch cultures, several indications were obtained which influenced our subsequent chemostat experiments (results not shown). First, the production of 987P fimbriae appeared to take place during the exponential growth phase. Second, when a fimbriate colony was used as inoculum no significant change in the percentage of fimbriate cells was observed during the different phases of growth. The percentage of fimbriate cells depended on the method used for analysis. The direct method of immunofluorescent staining of cells was preferred to the indirect method of analysis of colony morphology.

\section{Relation between specific growth rate and $987 P$ production}

Because of the dependence of the production of K99 fimbriae on the specific growth rate (van Verseveld et al., 1985) the effect of specific growth rate on phase variation and 987P production was examined. To reliably assess effects of growth conditions on production, one must be sure that the state of the culture is representative of particular growth circumstances. In general, a chemostat culture is assumed to be in a steady state after a minimum of six generations of growth under unaltered circumstances. Because phase variation is random (Brinton, 1959), it was important to establish whether or not cultures could be obtained that were in a steady state with respect to phase variation. Fig. 1 illustrates two important characteristics of production of $987 \mathrm{P}$ fimbriae concerning this point. First, the effect of growth for an extended period of time in the chemostat is seen. The production of fimbriae in the culture and the percentage of fimbriate cells remained constant during the fifty generations where no alterations were made in growth conditions. This suggests that fimbriae production per cell is constant at a constant growth rate. That the percentage of fimbriate cells remained constant can be explained either by an equilibrium in the phase variation of cells switching from $\mathrm{P}^{+} \rightarrow \mathrm{P}^{-}$and from $\mathrm{P}^{-} \rightarrow \mathrm{P}^{+}$, or because no phase variation was taking place at all. Second, this result also illustrates an effect of the specific growth rate on production of fimbriae and phase variation. After the specific growth 


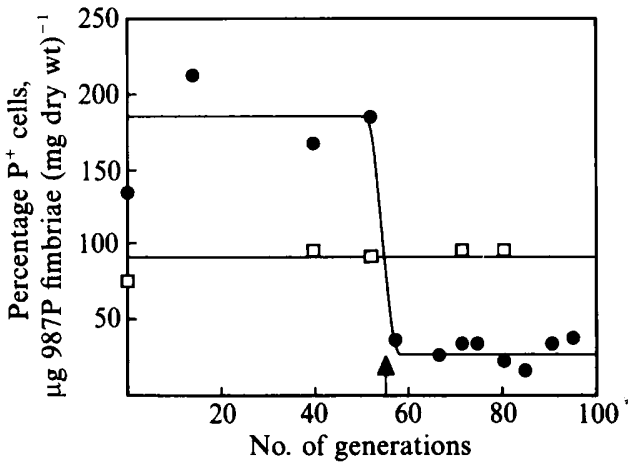

Fig. 1

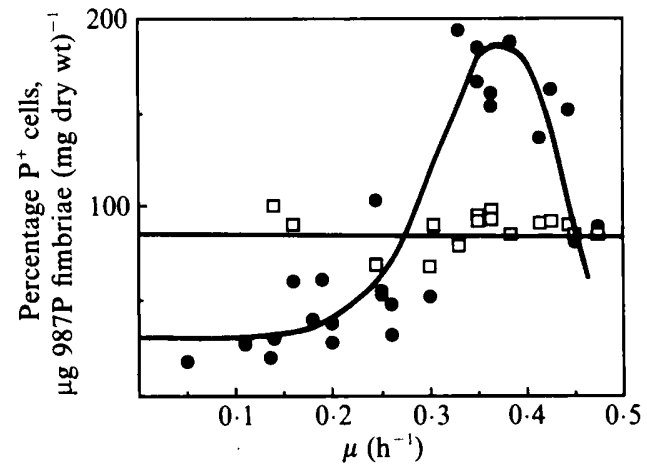

Fig. 2

Fig. 1. Production of 987P fimbriae $(O)$ and percentage of fimbriate $\left(\mathrm{P}^{+}\right)$cells $(\square)$ in a glucose-limited chemostat culture $(10 \mathrm{~mm})$ shifted from a high to a low specific growth rate under aerobic growth conditions at $37^{\circ} \mathrm{C}$. Production of fimbriae was followed over a large number of generations and is expressed per $\mathrm{mg}$ dry wt. The arrow indicates the switch from $\mu=0.35 \mathrm{~h}^{-1}$ to $\mu=0.14 \mathrm{~h}^{-1}$.

Fig. 2. Production of 987P fimbriae (O) and percentage of fimbriate cells $(\square)$ at various specific growth rates in steady state glucose-limited $(10 \mathrm{~mm})$ chemostat cultures grown under aerobic growth conditions at $37^{\circ} \mathrm{C}$. Production of fimbriae per $\mathrm{mg}$ dry wt is indicated for a $100 \%$ fimbriate culture (as calculated from the measured percentage of $\mathrm{P}^{+}$cells).

rate was lowered from $0 \cdot 35 \mathrm{~h}^{-1}$ to $0 \cdot 14 \mathrm{~h}^{-1}$, production of 987P fimbriae decreased within six generation times. As almost all cells still showed fluorescence, even though very much less so than at the higher growth rate, it seems that quantative regulation is influenced by the specific growth rate while phase variation is not.

On the basis of these results it was decided to examine the relation between the production of 987P fimbriae and the specific growth rate $(\mu)$ under aerobic growth conditions. The results are shown in Fig. 2. Both the measured percentage of fimbriate bacteria and the amount of fimbriae were as would be produced by a $100 \% \mathrm{P}^{+}$culture, representing the phase variation and the quantitative regulation respectively. The latter was calculated from the amount of fimbriae produced by the culture and the percentage of $\mathbf{P}^{+}$bacteria, assuming that fimbriate and nonfimbriate cells have the same dry weight per cell. Expressed in this manner, production of 987P fimbriae is independent of phase variation so different production levels indicate quantitative regulation. Again, no correlation was found between specific growth rate and the percentage of fimbriate cells. The percentage of $\mathrm{P}^{+}$cells was fairly constant (about $85 \%$ ) regardless of the specific growth rate (Fig. 2). At all specific growth rates fimbriae were produced. Production increased with increasing specific growth rate, with an apparent optimum at $\mu=0.40 \mathrm{~h}^{-1}$. Subsequently, the experiment was repeated under anaerobic growth conditions. Unfortunately, anaerobic growth of $E$. coli strain 1592 was poor on minimal medium with glucose ( $30 \mathrm{~mm})$. The maximum specific growth rate was low $\left(0 \cdot 16 \mathrm{~h}^{-1}\right)$. At a specific growth rate around $0.15 \mathrm{~h}^{-1}$ the glucose-limited cultures were $80 \% \mathrm{P}^{+}$and produced about $180 \mu \mathrm{g}$ of $987 \mathrm{P}$ fimbriae per $\mathrm{mg}$ dry wt, calculated for a $100 \%$ fimbriate culture. Addition of $0.01 \%$ yeast extract led to growth with a component of the yeast extract as the growth-limiting substrate. Passing oxygen over the culture was expected to enhance growth while maintaining an anaerobic environment in the culture. However, growth under these oxygen-limited conditions was still poor. Production of fimbriae under these latter two circumstances was of the same order of magnitude as for anaerobic cultures growing in minimal medium; the percentage of $\mathrm{P}^{+}$cells in these cultures was $70-90 \%$.

\section{Effect of medium composition on phase variation}

Preliminary experiments using batch cultures in different types of media indicated an effect of medium composition on phase variation. In these experiments it was noted that growth on minimal medium always resulted in a bacterial population showing agglutination with $987 \mathrm{P}$ - 


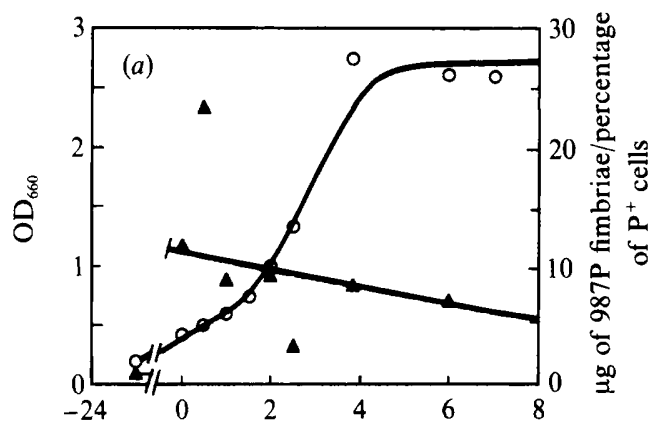

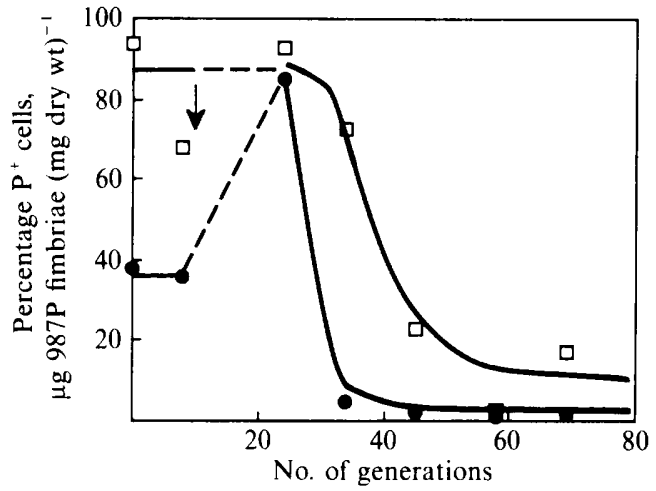

Fig. 3

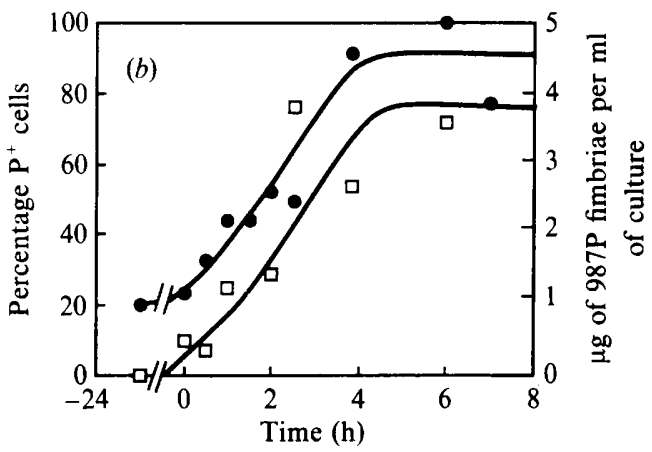

Fig. 4

Fig. 3. Production of $987 \mathrm{P}$ fimbriae $(\odot)$ and phase variation $(\square)$ of $E$. coli in a chemostat culture at a growth rate of $0.32 \mathrm{~h}^{-1}$ on different media under aerobic growth conditions at $37^{\circ} \mathrm{C}$. After eight generations growth on minimal medium with glucose $(10 \mathrm{mM})$ the medium inflow was changed to TSB $\left(6 \mathrm{~g} \mathrm{l}^{-1}\right)$; the arrow indicates the change. The dotted lines indicate the period of time in which the culture was not in a steady state due to the change in growth medium.

Fig. 4. Production of 987P fimbriae and percentage of fimbriate cells in an E. coli batch culture in minimal medium with glucose $(10 \mathrm{mM})$ under aerobic growth conditions at $37^{\circ} \mathrm{C}$. The culture was inoculated at $-24 \mathrm{~h}$ with washed cells from the stationary phase of a batch culture on TSB. (a) Growth curve $(O)$ and production of $987 \mathrm{P}$ fimbriae per $\mathrm{P}^{+}$fraction $(\boldsymbol{A})$. This was calculated by dividing the amount of fimbriae per $\mathrm{OD}_{660}$ unit by the fraction of $\mathrm{P}^{+}$bacteria. These two values, the amount of fimbriae per $\mathrm{OD}_{660}$ unit and the percentage of $\mathrm{P}^{+}$cells $(\square)$, are shown in $(b)$.

specific agglutinating antiserum, while growth on Minca medium with yeast extract did so in approximately $60 \%$ of cases.

It is possible to study the $\mathrm{P}^{+} \rightarrow \mathrm{P}^{-}$switch due to changes in medium composition in a chemostat by starting with a culture growing on minimal medium, this being $50-80 \% \mathrm{P}^{+}$. fimbriate. Fig. 3 shows the effect of a change from minimal to rich medium on production of fimbriae and on the percentage of fimbriate cells. During the experiment a constant dilution rate was maintained $\left(0.32 \mathrm{~h}^{-1}\right)$. This implies that a constant specific growth rate was maintained at constant biomass concentration with no other changes in growth circumstances. After 12 generations of growth on minimal medium with glucose as growth-limiting substrate, TSB, in which glucose is also the growth-limiting substrate, was supplied to the culture. This resulted in an initial enrichment of the growth medium. After a minimum of six generations the culture can be considered to be growing on TSB with the original specific growth rate. In Fig. 3 the transition time is indicated by dotted lines. After a total of 24 generation times, that is 12 generations after the switch, the observed changes can be attributed to the effect of growth on TSB. The concentration of fimbriae dropped strongly within ten generation times indicating a dilution of previously synthesized fimbriae. A reduction in the percentage of fimbriate cells was 
Table 1. Frequency of phase variation by the 987 P-fimbriae-producing strain of E. coli 1592

\begin{tabular}{|c|c|c|}
\hline \multicolumn{3}{|c|}{$\begin{array}{l}\text { Frequency of phase variation was calculated as described by Eisenstein (1981). The } \\
\text { generations) was } 25-26 \text { for grey colonies and } 27-28 \text { for white colonies. } M \text {, no. of cells } t \\
\text { phase; } N \text {, total no. of cells. }\end{array}$} \\
\hline $\begin{array}{l}\text { Colony morphology } \\
\text { of starting material }\end{array}$ & $M / N$ & Frequency \\
\hline Grey $\left(\mathrm{P}^{+}\right)$ & $\begin{array}{l}107 / 308 \\
217 / 234 \\
354 / 538 \\
403 / 604\end{array}$ & $\begin{array}{c}\mathrm{P}^{+} \rightarrow \mathrm{P}^{-} \\
1.31 \times 10^{-2} \\
3.64 \times 10^{-2} \\
2.56 \times 10^{-2} \\
2.57 \times 10^{-2}\end{array}$ \\
\hline & Mean $\pm S D$ & $2.52 \pm 0.95 \times 10^{-2}$ \\
\hline White $\left(\mathrm{P}^{-}\right)$ & $\begin{array}{r}5 / 856 \\
14 / 808 \\
3 / 296 \\
22 / 681\end{array}$ & $\begin{array}{c}\mathrm{P}^{-} \rightarrow \mathrm{P}^{+} \\
2.16 \times 10^{-4} \\
6.42 \times 10^{-4} \\
3.62 \times 10^{-4} \\
1.19 \times 10^{-3}\end{array}$ \\
\hline & Mean $\pm S D$ & $4.07 \pm 2 \cdot 16 \times 10^{-4}$ \\
\hline
\end{tabular}

also observed but the change in phase variation took place at a slower rate: during 34 generation times the percentage of positive cells decreased from $93 \%$ to $2.5 \%$. If a phase switch in only one direction is assumed due to the specific growth circumstances, the frequency of the $\mathrm{P}^{+} \rightarrow \mathrm{P}^{-}$ phase variation can be calculated by the formula $(M / N) / g$ in which $M / N$ is the fraction of the total bacterial population which has changed phase in $g$ generation times (Enomoto \& Stocker, 1975). In this experiment $M / N$ is measured as the difference in percentage of $\mathrm{P}^{+}$cells. In the $\mathrm{P}^{+} \rightarrow \mathrm{P}^{-}$direction the calculated frequency of phase variation was $2.7 \times 10^{-2}$ per generation.

Because the lag phase of strain 1592 for growth on minimal medium is extremely long, it was not possible to use the reverse of the experiment described above to examine the $\mathrm{P}^{-} \rightarrow \mathrm{P}^{+}$switch under influence of medium composition. Instead the following approach was used. From a single TSB-grown, agglutination-negative colony, $100 \mathrm{ml}$ TSB was inoculated. After the stationary phase was reached cells were harvested, washed and used to inoculate $100 \mathrm{ml}$ of minimal medium to an $\mathrm{OD}_{660}$ of approximately $0 \cdot 2$. Samples were taken as soon as growth started which was almost $24 \mathrm{~h}$ after inoculation of the minimal medium (Fig. 4). As the culture started to grow in minimal medium the percentage of $\mathrm{P}^{+}$cells gradually increased, as did the amount of fimbriae (Fig $4 b$ ). The calculated amount of fimbriae produced per cell in the positive phase remained fairly constant (Fig. $4 a$ ). Again, assuming that phase variation is taking place in one direction only, the frequency of the phase variation can be calculated. In this case two alternatives for $g$, the number of generations, can be taken for the calculation. First, $g$ can be considered as the number of generations of actual growth. Thus, using the $t=0$ and $t=4$ measurements, the frequency is $1.4 \times 10^{-1}$. If the lag phase is taken into consideration as well, $g$ becomes much higher. Maintaining $1.25 \mathrm{~h}$ as the mean generation time, $g$ is 19 . The frequency of phase variation using this value becomes $2.9 \times 10^{-2}$.

The frequencies of variation for the switch between the 987P fimbriate and non-fimbriate phase were in the same order of magnitude as found for the $\mathrm{P}^{+} \rightarrow \mathrm{P}^{-}$switch in the expression of S- and type 1 fimbriae using similar analysis methods (Nowicki et al., 1985). Higher frequencies or phase variation of type 1 fimbriae were described by Eisenstein (1981). However, both the growth conditions and the analytical methods used in the experiments described above for $987 \mathrm{P}$ fimbriae differed appreciably from the method used by Eisenstein (1981). As a comparison, the frequency of variation was also measured using the latter method, which is based on the analysis of the phase switch taking place during outgrowth of a single cell to a colony. Colony morphology is used to determine the state of the original single cell: The results are shown in Table 1 . The frequency of the switch $\mathrm{P}^{+} \rightarrow \mathrm{P}^{-}$, determined by analysing a grey colony, was 50 to 100 times higher than the switch frequency $\mathrm{P}^{-} \rightarrow \mathrm{P}^{+}$. 


\section{DISCUSSION}

The experiments showed that the amount of 987P fimbriae produced per cell, as well as the phase variation of individual cells, was subject to physiological control. The amount of $987 \mathrm{P}$ antigen produced per cell in the positive phase $\left(\mathrm{P}^{+}\right)$was dependent on the specific growth rate $(\mu)$ and is optimal at $\mu=0.40 \mathrm{~h}^{-1}$ under aerobic growth conditions. These findings are in contrast with the quantitative regulation found for K 99 and $\mathrm{F} 41$ production by $E$. coli. The latter are also increasingly produced with higher specific growth rates, but the production is optimal at $\mu_{\max }$ (van Verseveld et al., 1985). The maximal production of 987P fimbriae under anaerobic and oxygen-limited conditions was comparable to the maximal production under aerobic conditions and also takes place close to $\mu_{\max }$. However, under anaerobic conditions the $\mu_{\max }$ was only $0 \cdot 16 \mathrm{~h}^{-1}$, so at low specific growth rates the production of $987 \mathrm{P}$ fimbriae was higher under anaerobic than under aerobic conditions. Also, these results are in contrast with those obtained for production of $\mathrm{K} 99$ and F41 fimbriae. These showed the same dependence on growth rate under anaerobic as under aerobic growth conditions (van Verseveld et al., 1985). These findings suggest that the quantitative regulation of 987P fimbriae synthesis is different to that for other fimbriae produced by enterotoxigenic $E$. coli strains. Neither physiological nor genetic mechanism of regulation can be deduced from the experiments described in this paper. The percentage of $\mathrm{P}^{+}$cells remained more or less constant at different growth rates. The variations found in the percentage of $\mathrm{P}^{+}$cells between different chemostat runs most likely do not represent phase variation, but are inherent to the analysis methods used. A similar variation was found for the production of $987 \mathrm{P}$ fimbriae. This contrasts with the amount of fimbriae produced per cell in the positive phase, indicating that quantitative physiological regulation and phase variation are two independently operating mechanisms influencing the amount of fimbriae produced by a cell.

After a change in nutrients from a minimal medium with glucose to TSB with glucose, the amount of fimbriae in the culture decreased, but only after the culture was growing on TSB. The nature of the growth-limiting substrate, glucose, remains the same, so the reduction of $987 \mathrm{P}$ fimbriae synthesis must be attributed to an additional limitation in a particular nutrient, or to the presence of some component(s) in TSB. The reduction in the percentage of $\mathrm{P}^{+}$bacteria in this experiment was slower than expected from dilution of the fimbriated culture. Apparently, growth on TSB affects both quantitative and qualitative regulation. A decrease in production of fimbriae in complex media has also been observed for K99 fimbriae and was due to the presence of alanine (de Graaf et al., 1980).

We assume that qualitative regulation based on phase changes takes place under all growth conditions, and that the percentage of cells in the positive phase represents the result of a net balance between $\mathrm{P}^{+} \rightarrow \mathrm{P}^{-}$and $\mathrm{P}^{-} \rightarrow \mathrm{P}^{+}$switches. The large differences in the percentage of $\mathrm{P}^{+}$ cells measured in cultures growing in minimal medium with glucose or on TSB demonstrate that this balance is affected by environmental factors. This change in balance can be caused either by a change in frequency of both switches or by a unidirectional change in either the $\mathrm{P}^{-} \rightarrow \mathrm{P}^{+}$or the $\mathrm{P}^{+} \rightarrow \mathrm{P}^{-}$switch. On basis of the experiments described here it is not possible to discriminate between these possibilities. It should be mentioned, however, that the calculated figures for all switch frequencies using the formula $(M / N) / g$ represent the frequency of a unidirectional switch.

For the $\mathrm{P}^{-} \rightarrow \mathrm{P}^{+}$switch induced by growth on minimal medium two frequencies have been given depending on the value used for $g$ in the calculations. The phase switch is due to a genetic event which may not be dependent on growth and may occur during the adaptation of the cells to their new environment. Therefore, we prefer to use the value for $g$ which takes into account the long lag phase of cells switched from TSB to minimal medium. Based on this $g$ value the $\mathrm{P}^{-} \rightarrow \mathrm{P}^{+}$ frequency was calculated to be $2.9 \times 10^{-2}$. The frequency of the $\mathrm{P}^{+} \rightarrow \mathrm{P}^{-}$switch induced by growth on TSB in the chemostat was $2.7 \times 10^{-2}$.

Using a different method for the determination of switch frequencies (Eisenstein, 1981) and growing the cells on solid instead of liquid media, the frequencies were $2.52 \times 10^{-2}$ for $\mathrm{P}^{+} \rightarrow \mathrm{P}^{-}$ and $4.07 \times 10^{-4}$ for $\mathrm{P}^{-} \rightarrow \mathrm{P}^{+}$. The difference between these values and the ones mentioned above could be due to different growth conditions. The latter method has the advantage that both frequencies concern phase variation taking place under identical growth conditions, but is less 
suited for calculations using the formula $(M / N) / g$. In this formula a linear relationship is assumed between $M / N$ and $g$. It is more likely, however, that the actual number of cells switching phase $(M / N)$ is dependent on the relative sizes of the population of cells in a particular phase. Because the latter changes constantly until a new equilibrium is reached, so will $M / N$. By analysing colonies it is not possible to determine if a new equilibrium has been reached during the number of generations growth $(\mathrm{g})$ is allowed. The best approach to study which physiological factors influence phase variation, seems to be to determine the factors that induce change, both to analyse the resulting equilibrium situations and to calculate the rate at which these changes take place. For this, experiments of the type described for liquid cultures, combined with a direct analysis of the cells in the culture seem to be most suited.

One way to enrich a culture for cells in the fimbriate phase is to let growth take place in stationary liquid medium after which a pellicle of fimbriate bacteria will form at the surface of the liquid (Brinton, 1965, Old \& Duguid, 1970). The results of the anaerobic and oxygen-limited chemostat in which a high production of fimbriae and a high percentage of $\mathrm{P}^{+}$cells were found, suggest that the type of metabolism during anaerobic growth is important for synthesis of fimbriae. Enrichment of $987 \mathrm{P}^{+}$cells in static cultures could then be explained not by outgrowth at the culture surface but by anaerobic growth. Brinton (1965) has suggested that pellicle formation is caused by fimbriate cells having a growth advantage over non-fimbriate cells under anaerobic conditions. The type of experiments described here are not suitable to determine if this is this case, but the results do not exclude this possibility. If mutants could be obtained in which the phase variation is locked in one state, it would be possible to determine if there are any physiological differences between $\mathrm{P}^{+}$and $\mathrm{P}^{-}$bacteria.

The amount of fimbriae produced by cells in the positive phase was dependent on the growth conditions, but was always larger than the amount produced by cells in the negative phase. This suggests that the cell can adapt synthesis of fimbriae over a large range by combining both regulatory systems. By doing this the cell can adapt fimbria production to environmental changes of short duration by quantitative regulation and to changes of a more permanent nature by qualitative regulation, as encountered in the in vivo situation.

This investigation was supported by the Netherlands Foundation for Biological Research (BION) with financial aid from the Netherlands Organization for Scientific Research (NWO).

\section{REFERENCES}

Abraham, J. M., Freitag, C. S., Clements, J. R. \& EISENSTEIN, B. I. (1985). An invertible element of DNA controls phase variation of type I fimbriae of Escherichia coli. Proceedings of the National Academy of Sciences of the United States 82, 5724-5727.

Brinton, C. C., JR (1959). Non-flagellar appendages of bacteria. Nature, London 183, 782-786.

Brinton, C. C. (1965). The structure, function, synthesis and genetic control of bacterial pili and a molecular model for DNA and RNA transport in Gram negative bacteria. Transactions of the New York Academy of Sciences 27, 1003-1054.

Dorman, C. J. \& Higgins, C. F. (1987). Fimbrial phase variation in Escherichia coli: dependence on integration host factor and homologies with other site-specific recombinases. Journal of Bacteriology 169, 3840-3843.

Eisenstein, B. I. (1981). Phase variation of type I fimbriae in Escherichia coli is under transcriptional control. Science 214, 337-339.

Eisenstein, B. I., Sweet, D. S., Vaughn, V. \& FRIEDMAN, D. I. (1987). Integration host factor is required for the DNA inversion that controls phase variation in Escherichia coli. Proceedings of the National Academy of Sciences of the United States 84, 6506-6510.
Enomoto, M. \& Stocker, B. A. D. (1975). Integration, at $H A G$ or elsewhere, of $H 2$ (phase-2 flagellin) genes transduced from Salmonella to Escherichia coli. Genetics 81, 595-614.

GaAstra, W. \& dE GraAF, F. K. (1982). Host-specific fimbrial adhesins of noninvasive enteroxigenic Escherichia coli strains. Microbiological Reviews 46, 129-161.

de Graaf, F. K. \& KlaAsen, P. (1986). Organization and expression of genes involved in the biosynthesis of 987P fimbriae. Molecular and General Genetics 204, 75-81.

de Graaf, F. K. \& KlaAsen, P. (1987). Nucleotide sequence of the gene encoding the $987 \mathrm{P}$ fimbrial subunit of Escherichia coli. FEMS Microbiology Letters 42, 253-258.

DE GraAF, F. K. \& Moor, F. R. (1986). The fimbrial adhesins of Escherichia coli. Advances in Microbial Physiology 28, 65-143.

de Graaf, F. K., Klaasen-Boor, P. \& van Hees, J. E. (1980). Biosynthesis of the K99 surface antigen is repressed by alanine. Infection and Immunity 30, 125128.

Guinee, P. A. M., Jansen, W. M. \& Agterberg, C. M. (1976). Detection of the K99 antigen by means of agglutination and immunoelectrophoresis in 
Escherichia coli isolates from calves and its correlation with enterotoxigenicity. Infection and Immunity 13, 1369-1377.

KLEMM, P. (1986). Two regulatory fim genes, fim $B$ and fim $E$, control the phase variation of type I fimbriae in Escherichia coli. EMBO Journal 5, 1389-1393.

McKinney, R. M., Spillane, J. T. \& Pearce, G. W. (1986). A simple method for determining the labelling efficiency of fluorescein isothiocyanate products. Analytical Biochemstry 14, 421-428.

Nagy, B., Moon, H. W. \& Isaacson, R. E. (1976). Colonization of porcine small intestine by Escherichia coli: ileal colonization and adhesion by pig enteropathogens that lack K 88 antigen and by some acapsular mutants. Infection and Immunity 13, 1214 1220.

NAGY, B., MoON, H. W. \& IsaAcson, R. E. (1977). Colonization of porcine intestine by Escherichia coli: selection of piliated forms in vivo, adhesion of piliated forms to epithelial cells in vitro, and incidence of pilus antigen among porcine enteropathogenic Escherichia coli. Infection and Immunity 16, 344-352.
Nowicki, B., Rhen, M., VÄISÄNEN-Rhen, V., Pere, A. \& KORHONEN, T. K. (1984). Immunofluorescence study of fimbrial phase variation in Escherichia coli KS71. Journal of Bacteriology 160, 691-695.

Nowicki, B., RHEN, M., VÄIsÄnen-Rhen, V., PERE, A. \& Korhonen, T. K. (1985). Kinetics of phase variation between $\mathrm{S}$ and type 1 fimbriae of Escherichia coli. FEMS Microbiology Letters 28, 237-242.

Old, D. C. \& Duguid, J. P. (1970). Selective outgrowth of fimbriate bacteria in static liquid medium. Journal of Bacteriology 103, 447-456.

Stouthamer, A. H. \& Bettenhausen, C. W. (1975). Determination of the efficiency of oxidative phosphorylation of continuous cultures of Aerobacter aerogenes. Archives of Microbiology 102, 187-192.

VAN VERSEVELD, H. W., BAKKeR, P., VAN DER Woude, T., Terleth, C. \& DE GraAf, F. K. (1985) Production of fimbrial adhesins K99 and F4l by enterotoxigenic Escherichia coli as a function of growth-rate domain. Infection and Immunity 49, 159163. 